\title{
Early Stage Screening for Ovarian Cancer: The Case for Biosensor Technology
}

\author{
Michael Thompson* \\ Department of Chemistry, University of Toronto, 80 St George Street, Toronto, ON M5S 3H6, Canada
}

*Corresponding author: Michael Thompson, Department of Chemistry, University of Toronto, 80 St. George Street, Toronto, ON M5S 3H6, Canada; E-mail: m.thompson@utoronto.ca

Received: 17 Aug, 2020 | Accepted: 28 Aug, 2020 | Published: 02 Sep, 2020

Citation: Thompson M (2020) Early Stage Screening for Ovarian Cancer: The Case for Biosensor Technology. J Breast Cancer Res Adv 2(1): dx.doi.org/10.16966/2638-3527.109

Copyright: (c) 2020 Thompson M. This is an open-access article distributed under the terms of the Creative Commons Attribution License, which permits unrestricted use, distribution, and reproduction in any medium, provided the original author and source are credited.

\section{Editorial}

Malignant ovarian Cancer (OC) is the deadliest gynaecological cancer and the sixth most common cause of cancer-related death. The fact that the 5 -year survival rate for OC detected at stage 1 is over $90 \%$, leads to the clear conclusion that patient assessment at this level would be highly desirable. Indeed, a 2016 report [1] on the disease produced the recommendation, "Researchers and funding organizations should focus on the development and assessment of early detection strategies that extend beyond current imaging modalities and biomarkers and that reflect the pathobiology of each ovarian cancer subgroup." Recognized issues with the existing CA125 assay have spawned significant interest in alternative biomarkers for OC. Among these are HE4, osteopontin, HSP-27 HSP-60, HSP10, RAGE, CA15-3 (MUC1), calreticulin vimentin and fibrinogen- $\gamma$, mesothelin, and LPA [2]. There has also been FDA approval of a very limited number of screening panels such as, for example, OVA1 (CA 125-II, HE4, apolipoprotein A-1, FSH, and transferin). The ideal detection configuration for such markers would be one that is capable of measurement at the concentration characterized for early stage presence, operation in blood or serum, and in multiplexed (multianalyte) fashion. Furthermore, it is advantageous if the protocol for assay can generate information with respect to cell line(s). For example, there are 5 types of ovarian epithelial carcinoma with the high-grade serous version representing $75 \%$ of cases. (It is worth noting that OC also occasionally involves the BRAC1 and BRAC2 genes associated with breast cancer).

In order to assay the concentration of markers mentioned above, it is evident that sophisticated instrumentation such as liquid chromatography-mass spectrometry technology employed in a multi-step method can effectively produce such measurements. However, the approach is expensive and time-consuming to operate especially on large numbers of samples. Current detection through radioimmunoassay, ELISA and various luminescence methods offer sensitivity, precision and selectivity, but these techniques also suffer from several drawbacks such as long test times, high costs, and the need for qualified personnel and sophisticated instrumentation to perform the assays. In contrast, as we have pointed out with regard to OC markers, biosensor technology provides the possibility for direct conversion of the presence (and concentration) of a marker into a sensor -generated electrical signal [3]. This type of device is capable of function in relatively small samples, in a flow-injection protocol, provision of rapid signal generation, inexpensive manner, and, importantly, multiplexed detection. It is envisaged that two distinct categories of application of these devices are available, that is their use in the central clinical biochemistry facility or in a point-of-care fashion. Clearly, cost-effective, general screening of sera in the former would be appropriate for employment of these sensors, especially given the usual robot-oriented, automated equipment available in such a facility.

The key ingredients required for biosensor function in this type of application are a device-attached probe for selective interaction with marker(s), a sensitive sensor with appropriate read-out, and, critically the capability to operate in human serum without interfacial interference from other components of the biological fluid. A limited number of probes have seen development for OC markers; in our case we have employed gelsolin for LPA and aptamers for HSP-10 [4]. However, it is clear that much remains to be accomplished in this area, which may well include, for example, advances in immunochemical species. In terms of devices for marker detection, various forms of electrochemical (eg: electrical impedance spectroscopic), acoustic wave (ultra-high frequency shear wave sensors) and optical (surface plasmon resonance) systems are available. The first two of these techniques are eminently capable of being used for multianalyte assay in that, via photolithography, MEMS technology and the like, a large number of probes sites can be interrogated. This approach could lead to the "holy grail" of being able to perform a true precision medicine style appraisal of a particular patient sample. Finally, it is clear that the device-probe configuration must detect markers at anticipated low concentrations in the analytically-difficult medium, serum. In our case we have employed surface modification with an anti-fouling agent in order to successfully reduce interfacial interference, especially 
for detection of the breast metastasis marker, PTHrP, achieved in a sister study [5].

\section{Conclusion}

Biosensor technology provides potential for the production of clinical high throughput detection of OC markers in a costeffective, multiplexed fashion. Given the well-characterized high survival rate observed for early-stage diagnosis, the technology offers the possibility to screen a large patient population resulting in the exciting result of significant reduction of incidence of the disease into later stages.

\section{References}

1. Committee on the State of the Science in Ovarian Cancer Research; Board on Health Care Services; Institute of Medicine; National Academies of Sciences, Engineering, et al. (2016) Ovarian Cancers:
Evolving Paradigms in Research and Care. National Academies Press, Washington (DC).

2. Montagnana $M$, Benati $M$, Danese $E$ (2017) Circulating biomarkers in epithelial ovarian cancer diagnosis: from present to future perspective. Ann Transl Med 5: 276.

3. De La Franier B, Thompson M (2019) Early stage detection and screening of ovarian cancer: A research opportunity and significant challenge for biosensor technology. Biosens Bioelectron 135: 71-81.

4. De La Franier B, Thompson M (2020) Detection of the Ovarian Cancer Biomarker Lysophosphatidic Acid in Serum. Biosensors (Basel) 10: 13.

5. Crivianu-Gaita V, Aamer M, Posaratnanathan RT, Romaschin A, Thompson M (2016) Acoustic wave biosensor for the detection of the breast and prostate cancer metastasis biomarker protein PTHrP. Biosens Bioelectron 78: 92-99. 